\title{
Relationship between DASH Questionnaire and Objective Variables in Carpometacarpal Joint Osteoarthritis
}

\author{
${ }^{1}$ Tecan Hand Clinic Center, University of Málaga, Spain \\ ${ }^{2}$ Phisical Therapy Departament, University of Málaga, Spain \\ ${ }^{3}$ Physical Therapy Section - Faculty of Health Sciences - University of Malaga, Spain \\ ${ }^{4}$ Moviment $i$ Salut Center, Valencia, Spain \\ ${ }^{5}$ Comarcal Hospital Axarquía, Vélez, Spain
}

Cantero-Téllez Raquel1,2*, Medina-Porqueres Ivan ${ }^{3}$, Such-Sanz Arturo ${ }^{4}$, García-Orza Santiago ${ }^{5}$ and Martín-Valero Rocío

\begin{abstract}
Study background: Carpometacarpal joint osteoarthritis has an important functional consequence on Activity daily life. Pain, is the main reason for medical consultation described in the literature. Last years, outcomes based on self-report of function and patient satisfaction have become variables increasingly important for measurement improvement.
\end{abstract}

Objective: Determinate the relationship between subjective variables (pain and function) and pinch and grip force in patients suffering from carpometacarpal joint osteoarthritis.

Methods: Observational analytic design. Final sample consisted of 72 patients diagnosis of carpometacarpal joint osteoarthritis, grade 2/3 Eaton. Pain, Grip and pinch force were measured and compared to patient-report functional outcomes (DASH)

Results: For function, the most significant model $(R 2=0.89)$ included grip, key, tip to tip and 3 points pinch. But it is tip to tip pinch force which has a stronger relationship with DASH (Standardized B: -736) questionnaire.

Conclusion: Function referred by the patient depends on pinch, grip force and pain, but is tip to tip pinch force the best predictor of function in a lineal correlation.

Keywords: Carpometacarpal joint; Function; Hand; Thumb pain; Osteoarthritis; Outcome measurements; Rhizartrosis

\section{Introduction}

Degenerative thumb process have an important functional consequences due to compensatory movements that have been unbalanced the musculoskeletal system during the development of the disease, causing pain and decreased mobility [1].

Joints typically affected in hand degenerative process are usually the interphalangeal and metacarpophalangeal joint with particular impact on the thumb, which is the key to understanding most of hand functional movements. Osteoarthritis (OA) of carpometacarpal (CMC) joint of the thumb is being the most reported painful joint compared with all other hand OA pain [2]. This pain imposes substantial restrictions on everyday task. This can affect how well the thumb moves, how strong a person's grip is, and how well a person can perform routine at home or at work. Opposition, is the most important hand functional movement, and requires stability in order to get a power pinch and grip. Nevertheless, thumb strength and position used during functional tasks, also depends on the weight of the object to be held, on its size, shape and material $[3,4]$, so that, also depends on how the person can use the thumb. These facts must be taken into consideration during hand rehabilitation program.

Clinical symptoms of degenerative CMC joint include pain, stiffness and weakness. This symptom appears when ligamentous laxity structures can't continue performing its function, and develop when ligamentous laxity at the trapezium metacarpal joint causes dorsovolar translation and recurrent synovitis [2,5] with a loss of grip, strength and mobility deficits. This loss of function is reflected in the difficulty patients to perform ADL (Activity daily life) [2]. Pain, is the main reason for medical consultation and the most influential factor that prevent them from performing activities like writing, gardening, turning on a tap, opening jars, using scissors and, ultimately, to bring out everyday tasks. In fact, pain is the main objective described in the literature in the treatment of this pathology, as it is the most influential factor in function according to some authors [2,6,7]. Some studies confirm that decreasing pain in degenerative hand process improve function and patient satisfaction. However, when quantifying the results of conservative and surgical treatments for degenerative thumb, objective parameters such as range of motion (ROM), strength, and radiological findings are used to quantify the outcome of the intervention [8]. Despite improving parameters such as strength and mobility, several studies have shown that these variables do not necessarily correlate with the patient's perception of treatment success. Several researchers have described the discrepancy between assessments of clinical and functional outcomes in degenerative hand [2,7]. Last years, results based on self-assessment of function, activities of daily living (ADL), quality of life and patient satisfaction have become variables increasingly important, but its interpretation is complex, because of the variety of factors that influence the perception of a successful outcome.

*Corresponding author: Cantero-Téllez Raquel, PhD, University of Málaga, Spain, Tel: 0034-952226453; E-mail: cantero@uma.es

Received June 11, 2015; Accepted July 08, 2015; Published July 17, 2015

Citation: Cantero-Téllez R, Medina-Porqueres I, Such-Sanz A, Garcia-Orza S, Martin-Valero R (2015) Relationship between DASH Questionnaire and Objective Variables in Carpometacarpal Joint Osteoarthritis. J Arthritis S1: 004 doi:10.4172/2167-7921.S1-004

Copyright: @ 2015 Cantero-Tellez, et al. This is an open-access article distributed under the terms of the Creative Commons Attribution License, which permits unrestricted use, distribution, and reproduction in any medium, provided the original author and source are credited. 
Citation: Cantero-Téllez R, Medina-Porqueres I, Such-Sanz A, Garcia-Orza S, Martin-Valero R (2015) Relationship between DASH Questionnaire and Objective Variables in Carpometacarpal Joint Osteoarthritis. J Arthritis S1: 004. doi:10.4172/2167-7921.S1-004

Page 2 of 4

Methods and procedures for a proper clinical and functional thumb assessment are well described, but we don't know how each variable influences others. Main objective in our study is determinate the possible relationship between grip and pinch force, pain intensity and function in patient diagnosis of CMC joint osteoarthritis. Hypothetically, final results of the present study will allow us simplified evaluation and identify mean problems in order to prioritize goals of treatment for improving function in degenerative thumb process.

\section{Material and Method}

\section{Study designing}

Data were collected in Tecan Hand Center clinic in cooperation whit hand surgeons from University Hospital of Málaga between January and December 2014.

Main researcher was a hand therapy expert. When patient completed a self- administered DASH Spanish Version questionnaire and VAS questionnaire, the hand therapy performed grip $(\mathrm{Kg})$ and pinch measurement $(\mathrm{Kg})$. The intervention was normal practice; therefore no formal approval from ethics committee was necessary.

\section{Participants}

Patients diagnosis of carpometacarpal joint osteoarthritis, grade $2 / 3$ Eaton, previously confirmed by a hand surgeon radiologically were eligible for the study.

They were excluded if they had a neurological disorder affecting upper limb, had received previous treatment for this hand problem in the last 6 months including an intra-articular joint injection to wrist, fingers or thumb, fractures or significant injury or surgery to the wrist or hand, hand or finger tenosynovitis and Dupuytren's disease. Also excluded those who did not completed Dash Spanish version questionnaire. Final sample consisted of 72 patients (59 women and 13 men), with a mean age of 59,97 (SD 8,36).

\section{Instruments}

Grip strength was measured by Jamar dynamometer, and a pinch dynamometer was used for pinch strength. Patient was instructed to keep the shoulder adducted and neutrally rotated, the elbow flexed to $90^{\circ}$, with the wrist and forearm in a neutral position during test procedures.

Thumb pain was evaluated on a visual analogue scale (VAS) ranging from $0 \mathrm{~mm}=$ no pain to $100 \mathrm{~mm}=$ maximal pain. For information regarding patient estimates of their own function, we used Disability arm shoulder and hand questionnaire (DASH), Spanish Version, a self-administered questionnaire whit 30 questions. The DASH is an outcome tool designed to measure physical function and symptoms in persons with musculoskeletal disorders (MSDs) of the upper limb.

\section{Procedure}

All patients were evaluated by the same hand therapist, following the same protocol and using the same instruments, following recommendations of American Society of hand therapies (ASHT) [9].

First of all, patients were asked for completed DASH questionnaire and VAS scale.

For grip strength measurement a Jamar hydraulic dynamometer was used at the second position.

To determine pinch strength, tip to tip pinch (p-p), three points (3$\mathrm{p})$ and a key pinch (K-p) were measurement with a pinch dynamometer.

\section{Statistical analysis}

Before analyses data were tested for normality of the sample using Kolmogorov-Smirnov test.

In the first set of analyses, we explored the relationship between the variables measured in the study through correlational analyses. To this aim, Pearson correlations were employed.

The second set of analyses investigates through standard regressions the clinical predictors of subjective indexes of functionality, measured with DASH. Regression analyses were preferred to ANOVA because the former is better suited than the later when, as is the case in our study, there is unequal number of cases in cells, and the IV's are correlated. Additionally, continuous variables are better explored through the use of regression (in ANOVA continuous variables should be grouped what leads to a loss of information). For statistical analysis spss version 2.0 was used.

\section{Results}

Final sample consisted of a group of 72 people who had been diagnosed of CMC joint osteoarthritis, 59 women and 13 men with a mean age of $59,97 \pm 8,3$.

Table 1 summarizes mean and standard deviation values obtained by the participants in the variables measured.

As our primary goal was to analyse the relationships between DASH (Function) and objectives variables, a standard regression was run with grip, key, tip to tip and 3 points pinch as predictors and $\mathrm{DASH}$ as dependent variable. Analysis results a $\mathrm{R}=0,89(\mathrm{R} 2=0,79)$ $\mathrm{P}<0,001$. The model was a good predictor of function and it explained almost $79 \%$ percent of the variance in DASH. However, the analysis of the regression coefficients showed that only tip to tip pinch force (B standardized $-0,736(\mathrm{P}=0,00)$ arose as a valid predictor of DASH (Table 2).

\section{Discussion}

Findings confirm that there is a significant correlation between

\begin{tabular}{|c|c|c|}
\hline Variables & Means & SD \\
\hline DASH & 46,035 & 24,8261 \\
\hline grip & 15,257 & 9,7244 \\
\hline pp & 1,657 & 9140 \\
\hline Key & 2,192 & 8000 \\
\hline $3-P$ & 2,352 & 8817 \\
\hline VAS intensity & 4,60 & 2,330 \\
\hline
\end{tabular}

Table 1: Mean and standard deviation values obtained by the participants in the variables measured.

Grip: Grip force

P-P: Tip to Tip force

K-P: Key pinch force

3-P: 3 Points pinch force

\begin{tabular}{|c|c|c|}
\hline $\mathrm{R} 2=0,794(p=0,001)$ & Beta-S & Sig (P) \\
\hline &,- 101 &, 082 \\
\hline GRIP &,- 736 &, 000 \\
\hline K-P &,- 203 &, 093 \\
\hline 3-P &, 092 &, 210 \\
\hline
\end{tabular}

Table 2: More significant model using the outcome as the dependent variable in the DASH questionnaire.

P-P: Tip to Tip force

K-P: Key pinch force

3-P: 3 Points pinch force 
objectives and subjective variables. But also correlation between subjective variables (DASH score and pain) is significant, so there is a high significant correlation between pain and dysfunction. These two variables are closely related in cases of CMC joint osteoarthritis. We can confirm that for greater intensity of pain perceived by the patient, greater dysfunction reflected in the DASH questionnaire. But it can be considered a logical positive correlation because dash questionnaire includes pain intensity.

Nevertheless, relationship between pain and function could justifies the conclusions of the study of Dermid et al. [10], when he confirm that one of the primary objectives in the treatment of degenerative hand was reduction of pain, because this was the variable that most influenced in patients dysfunction. Other studies whit similar evidence regarding pain but based only on Pearson correlation results and in postoperative results include those of Wajon and Ada [12], Rogers and Wilder [13], Garfinkel et al. [14], Boustedt et al. [15], Thiele et al. [16], Weiss et al. [5] Day et al. [17], Micholvitz et al. [18], Kdzielski et al. [19], MacDermid et al. [20] and Goldfarb et al. [21]. Barthel et al. [11], also established a relationship between pain reduction and functional variables, finding that pain reduction had made an improvement in overall physical function and strength. Bijsterbosh et al. [22] also conducted a study to determine the potential impact of pain at the MCP and IF in osteoarthritis and dysfunction. They concluded that pain was the most influenced factor in the degree of dysfunction perceived by the patient. The study was conducted with a group of patients with a very specific problem in degenerative hand as our study, but they also arrived to this conclusion only whit the results of Pearson correlation.

Regarding the improvement of strength, between clinical measures significant correlations were found between key, tip to tip and 3 points pinch force (all p >.001). However these three variables, although approaching significance, did not correlate with grip force. We can't compared the results whit Barthel et al. conclusion [11], because although they confirm a improvement in force in relationship whit pain intensity, they don't made a difference between grip and three types of pinch. We can hypothesized that if there is an improvement in hand function, patient will used the hand in activity daily life and consequently pinch force will increase.

Regarding key pinch strength, in our results doesn't appear as a function predictor, but numerous previous studies where researchers want to assess the success of surgery in degenerative hand, use pinch strength as a "outcomes variables" [8]. In our study, the model that explained almost $80 \%$ percent of the variance in DASH $(F(4,67)=64,75$, $\mathrm{p}>$.001) included all clinical variables, but analysis of the regression coefficients showed that only tip to tip pinch force arose as a valid predictor of DASH and no key pinch force.

Nevertheless, studies who used key pinch force as a predictor of improvement doesn't used same measurements instruments. We have found only one investigation where the instrument used for measurement key pinch strength was the same as we used (Jamar hydraulic dynamometer). So we can't establish comparison with other studies in order to reach a conclusion where the assessment instrument used was different.

Others authors have found in their studies relationships between force and function. Bain et al. [23], Mandl et al. [24], Goldfarb et al. [21] or MarcDermid et al. [20], establishing a relationship between pinch strength, range of movement and degree of dysfunction described by the patient, but in these studies, we can see that there is a range in correlation coefficients ranging from 0.11 to 0.71 for strength and from 0.19 to 0.6 for the range of movement. Conclusions were reached considering only the degree of correlation of different variables. We have also found this correlation in our results but in the regression analysis, tip to tip pinch strength is the only one predictor of clinical variables for hand dysfunction.

Deformity, is also introduced as a variable in research in rheumatic hand for different authors as Mandl et al. [24], which has detected a significant correlation between hand function and improvement of the physical appearance of the same after surgery, and Goldfarb et al. [21], who hypothesized in their result, the correction of the deformity of the CMC influences the degree of patient satisfaction and function. We have no introduce deformity a as a variable in our study because there is no an objective instrument for measurement thumb deformity describe in the literature. But we can hypothesize that there is a correction in the deformity of the CMC and function, because better alignment of the muscular structures allows the muscles to perform its function and improve the strength, or because the correct deformity decreases pain.

Future studies will be necessary for confirm these findings. An observational study were only one measurement is taken is no enough for conclude these relationship. Also, thumb biomechanical studies will help to understand the differences between pinch improvements. Maybe, the "key" could be not only pinch force, but also, orientation of first metacarpal respect trapezium in different types of pinch used in the activity daily life.

Findings confirm that there is a significant correlation between clinical measurements usually used in hand therapy as grip and pinch force, and functional outcomes perceived by the patient. Significant negative correlation had been found between pain and function. These two variables are closely related in cases of CMC joint osteoarthritis. In fact, DASH questionnaire includes the pain variable in some questions.

Tip to tip pinch force seems to be the best predictor of function, but we can't reach this conclusion because the statistical analysis performed in this study does not allow us to reach those conclusions. We can say, that there is a linear correlation between these two variables and that this correlation is positive.

Further studies with a deep statistical analysis will determine whether the conservative approach on the tip to tip pinch force could be considered the most important objective in patients suffering of carpometacarpal osteoarthritis in order to improve function perceived by the patient.

Study limitations: DASH questionnaire gives us information about global disability of the upper extremity. We have study a specific pathology of thumb, so that, we have to take into consideration that this questionnaire might not have sufficient specificity for this pathology. On the other hand, the statistical analysis we have used in this study, only gives us a linear correlation between variables, not the degree of influence this variables can have on hand function.

\section{References}

1. Kjeken I, Dagfinrud H, Slatkowsky-Christensen B, Mowinckel P, Uhlig T, et al. (2005) Activity limitations and participation restrictions in women with hand osteoarthritis: patients' descriptions and associations between dimensions of functioning. Ann Rheum Dis 64: 1633-1638.

2. Wajon A, Ada L (2005) No difference between two splint and exercise regimens for people with osteoarthritis of the thumb: a randomised controlled trial. Aust $\mathrm{J}$ Physiother 51: 245-249.

3. Kovler M, Lundon K, McKee N, Agur A (2004) The human first carpometacarpal joint: osteoarthritic degeneration and 3-dimensional modeling. J Hand Ther 17 $393-400$. 
Citation: Cantero-Téllez R, Medina-Porqueres I, Such-Sanz A, Garcia-Orza S, Martin-Valero R (2015) Relationship between DASH Questionnaire and Objective Variables in Carpometacarpal Joint Osteoarthritis. J Arthritis S1: 004. doi:10.4172/2167-7921.S1-004

4. Napier JR (1956) The prehensile movements of the human hand. J Bone Joint Surg Br 38-38B: 902-13.

5. Weiss S, Lastayo P, Mills A, Bramlet D (2004) Splinting the degenerative basal joint: custom-made or prefabricated neoprene? J Hand Ther 17: 401-406.

6. Poole JL, Watzlaf VJ, D'amico F (2004) A five-year followup of hand function and activities of daily living in systemic sclerosis (scleroderma). J Hand Ther 17: 407-411.

7. Michlovitz S, Hun L, Erasala GN, Hengehold DA, Weingand KW (2004) Continuous low-level heat wrap therapy is effective for treating wrist pain. Arch Phys Med Rehabil 85: 1409-1416.

8. Tägil M, Kopylov P (2002) Swanson versus APL arthroplasty in the treatment of osteoarthritis of the trapeziometacarpal joint: a prospective and randomized study in 26 patients. J Hand Surg Br 27: 452-456.

9. Clinical assessment and recommendations. American Society of Hand Therapists. 2nd ed. Chicago IL, USA.

10. MacDermid JC, Wessel J, Maclntyre N, Galea V (2008) The relationship between impairment, dexterity and self-reported disability of persons with osteoarthritis of the hand. J Hand Ther 21: 423-424.

11. Barthel HR, Peniston JH, Clark MB, Gold MS, Altman RD (2010) Correlation of pain relief with physical function in hand osteoarthritis: randomized controlled trial post hoc analysis. Arthritis Res Ther 12: R7.

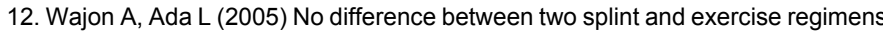
for people with osteoarthritis of the thumb: a randomised controlled trial. Aust $J$ Physiother 51: 245-249.

13. Rogers MW, Wilder FV (2007) The effects of strength training among persons with hand osteoarthritis: a two-year follow-up study. J Hand Ther 20: 244-249.

14. Garfinkel MS, Schumacher HR Jr, Husain A, Levy M, Reshetar RA (1994) Evaluation of a yoga based regimen for treatment of osteoarthritis of the hands. J Rheumatol 21: 2341-2343.
15. Boustedt C, Nordenskiöld U, Lundgren Nilsson A (2009) Effects of a handjoint protection programme with an addition of splinting and exercise: one year follow-up. Clin Rheumatol 28: 793-799.

16. Thiele J, Nimmo R, Rowell W, Quinn S, Jones G (2009) A randomized single blind crossover trial comparing leather and commercial wrist splints for treating chronic wrist pain in adults. BMC Musculoskelet Disord 10: 129

17. Day CS, Gelberman R, Patel AA, Vogt MT, Ditsios K, et al. (2004) Basal join osteoarthritis of the thumb: a prospective trial of steroid injection and splinting. J Hand Surg Am 29: 247-251.

18. Michlovitz S, Hun L, Erasala GN, Hengehold DA, Weingand KW (2004) Continuous low-level heat wrap therapy is effective for treating wrist pain. Arch Phys Med Rehabil 85: 1409-1416.

19. Kadzielski J, Malhotra LR, Zurakowski D, Lee SG, Jupiter JB, et al. (2008) Evaluation of preoperative expectations and patient satisfaction after carpal tunnel release. J Hand Surg Am 33: 1783-1788.

20. MacDermid JC, Roth JH, Rampersaud YR, Bain GI (2003) Trapezial arthroplasty with silicone rubber implantation for advanced osteoarthritis of the trapeziometacarpal joint of the thumb. Can J Surg 46: 103-110.

21. Goldfarb CA, Stern PJ (2003) Metacarpophalangeal joint arthroplasty in rheumatoid arthritis. A long-term assessment. J Bone Joint Surg Am 85-85A: 1869-78.

22. Bijsterbosch J, Visser W, Kroon HM, Stamm T, Meulenbelt I, et al. (2010) Thumb base involvement in symptomatic hand osteoarthritis is associated with more pain and functional disability. Ann Rheum Dis 69: 585-587.

23. Bain GI, Pugh DM, MacDermid JC, Roth JH (1995) Matched hemiresection interposition arthroplasty of the distal radioulnar joint. J Hand Surg Am 20: 944950

24. Mandl LA, Galvin DH, Bosch JP, George CC, Simmons BP, et al. (2002) Metacarpophalangeal arthroplasty in rheumatoid arthritis: what determines satisfaction with surgery? J Rheumatol 29: 2488-2491.
This article was originally published in a special issue, Arthritis \& Pain handled by Editor(s). Dr. Rostyslav $\vee$ Bubnov, Clinical Hospital Feofaniya 